\title{
Therapeutic Advances and Future Prospects in Progressive Forms of Multiple Sclerosis
}

\author{
Afsaneh Shirani $^{1}$ • Darin T. Okuda ${ }^{2}$ - Olaf Stüve ${ }^{1,3}$ \\ Published online: 4 January 2016 \\ (C) The American Society for Experimental NeuroTherapeutics, Inc. (outside the U.S.) 2015
}

\begin{abstract}
Identifying effective therapies for the treatment of progressive forms of multiple sclerosis (MS) is a highly relevant priority and one of the greatest challenges for the global MS community. Better understanding of the mechanisms involved in progression of the disease, novel trial designs, drug repurposing strategies, and new models of collaboration may assist in identifying effective therapies. In this review, we discuss various therapies under study in phase II or III trials, including antioxidants (idebenone); tyrosine kinase inhibitors (masitinib); sphingosine receptor modulators (siponimod); monoclonal antibodies (anti-leucine-rich repeat and immunoglobulin-like domain containing neurite outgrowth inhibitor receptor-interacting protein-1, natalizumab, ocrelizumab, intrathecal rituximab); hematopoetic stem cell therapy; statins and other possible neuroprotective agents (amiloride, riluzole, fluoxetine, oxcarbazepine); lithium; phosphodiesterase inhibitors (ibudilast); hormone-based therapies (adrenocorticotrophic hormone and erythropoietin); T-cell receptor peptide vaccine (NeuroVax); autologous T-cell immunotherapy
\end{abstract}

Olaf Stüve

olaf.stuve@utsouthwestern.edu

1 Department of Neurology and Neurotherapeutics, University of Texas Southwestern Medical Center, Dallas, TX 75390, USA

2 Department of Neurology and Neurotherapeutics, Clinical Center for Multiple Sclerosis, Multiple Sclerosis and Neuroimmunology Imaging Program, University of Texas Southwestern Medical Center, Dallas, TX 75390, USA

3 Neurology Section, VA North Texas Health Care System, Medical Service, Dallas VA Medical Center, Dallas, TX 75216, USA
(Tcelna); MIS416 (a microparticulate immune response modifier); dopamine antagonists (domperidone); and nutritional supplements, including lipoic acid, biotin, and sunphenon epigallocatechin-3-gallate (green tea extract). Given ongoing and planned clinical trial initiatives, and the largest ever focus of the global research community on progressive MS, future prospects for developing targeted therapeutics aimed at reducing disability in progressive forms of MS appear promising.

Keywords Multiple sclerosis · Progression · Primary progressive $\cdot$ Secondary progressive $\cdot$ Therapeutics $\cdot$ Clinical trials

\section{Introduction}

Multiple sclerosis (MS) is a chronic inflammatory, autoimmune, demyelinating, and neurodegenerative disease of the white and gray matter within the central nervous system (CNS) [1]. At diagnosis, approximately $85 \%$ of patients present with relapsing-remitting MS (RRMS), which is characterized by alternating episodes of neurological disability (relapses or attacks) followed by complete or partial recovery of symptoms [2]. About twothirds of patients diagnosed with RRMS will eventually make a transition to secondary progressive MS (SPMS) within 2 decades of onset [1]. However, about $10 \%$ of patients with MS present with primary progressive MS (PPMS), punctuated by gradually worsening neurological disability from symptom incipience with no distinct relapses or remissions [2]. The term "progressive MS" often refers to the combined population of patients with SPMS and PPMS. 
Although substantial progress has been made in the development of effective drug therapies for RRMS over the last 2 decades, as evidenced by the current 12 US Food and Drug Administration (FDA)-approved disease-modifying drugs for RRMS [3], no convincingly effective therapies exist for progressive forms of MS [4, 5]. This is while $>1$ million people (more than half of the 2.3 million population affected by MS worldwide) are living with a progressive form of MS [6]. Identifying effective therapies for progressive forms of MS represents a significant unmet need for patients, and a top priority for the global MS research community [7]. This challenging topic has recently attracted much attention, and increasing focus, as evidenced by the establishment in 2012 of the Progressive MS Alliance, the largest initiative aimed at speeding the developments of new treatments for progressive MS while overcoming existing research barriers $[8,9]$.

In the present article, we highlight the therapeutic implications based on known pathogenic mechanisms involved in MS, and the challenges of defining a progressive phenotype. We will then review prior unsuccessful trials for progressive MS followed by a detailed discussion of candidate therapeutics under investigation in phase II or III ongoing or planned trials. Finally, future directions for trials in progressive MS will be discussed.

\section{Insights into Pathogenesis and Therapeutic Implications}

The exact mechanism(s) of disease progression in MS remains unknown; however, our understanding of MS pathogenesis has significantly evolved over the last few decades with the advent of modern methodological tools in neuroimmunology, neurobiology, and neuroimaging. The various, and even conflicting, mechanisms that have been suggested to be involved in the pathogenesis of MS are outside the scope of this paper. A prevailing hypothesis involves a systemic adaptive inflammatory autoimmune process in the early stages that eventually results in the chronic activation of the CNS intrinsic innate immune system that ultimately leads to neurodegeneration [10]. While active demyelinating plaques associated with inflammation and blood-brain barrier (BBB) injury are the main neuropathological features of RRMS, in the progressive stages of the disease active neurodegeneration occurs on the background of mild-to-moderate inflammation. Major factors suggested to drive neurodegeneration include microglia activation and associated oxidative injury [11], resulting in mitochondrial insults and energy failure [12], and age-dependent iron accumulation in myelin and oligodendrocytes leading to oxidative tissue damage $[10$, 13].

The OLYMPUS trial strongly suggests a pathogenic role of B lymphocytes in some patients with early PPMS [14]. This notion is further substantiated by postmortem brain tissue from some patients with SPMS that demonstrated meningeal lymphoid follicles containing proliferating B cells [15]. While the presence of these lymphoid-like follicles has not been yet been reported in the meninges of PPMS brain tissue, diffuse T-cell and B-cell-driven meningeal inflammation has been shown, and was associated with a more severe clinical course, and more extensive demyelination and neurite loss in the cortical gray matter [16]. Interestingly, fingolimod, which failed to achieve its primary and secondary end points in a phase III clinical trial in patients with PPMS appears to increase the number of $\mathrm{B}$ cells in the cerebrospinal fluid (CSF) [17].

While the varying aspects of disease pathogenesis point to the potential benefit of a combination therapy approach (including anti-inflammatory, neuroregenerative/neurorehabilitative, and neuroprotective treatments) $[2,18]$, a key question that remains involves the potential existence of innate differences in the inflammatory response, if any, in relapsing versus progressive forms of MS given the failure of classic anti-inflammatory approaches in progressive MS [10].

\section{Progressive MS Phenotype}

While debates about whether PPMS and SPMS are part of the same disease spectrum or separate entities are still ongoing [19], an ever increasing amount of clinical and imaging evidence suggests that PPMS represents a spectrum of progressive MS phenotypes [20]. However, PPMS still remains a distinct course in the 2013 revision of defining the clinical course of MS because of the absence of acute attacks prior to clinical progression [20]; yet its pathological differences from SPMS are considered absolute rather than relative by some [21].

Natural history studies have shown that conversion to a secondary progressive course is the key determinant of long-term prognosis, independent of disease duration and early relapse frequency [22], and that disability worsening proceeds at a similar rate in SPMS and PPMS regardless of the initial disease course [23]. However, it has been shown that both pre- and post-progression relapses may accelerate time to disability milestones in progressive MS, a finding that implies possible beneficial effect of immunomodulatory therapies in patients with single-attack progressive MS and SPMS who have ongoing relapses [24].

It has recently been suggested that absence of a relapsing-remitting phase in patients with PPMS could be explained by lesions localizing in clinically silent regions of the CNS that cumulate to result eventually in physical disability. This is supported by findings from the long-term follow-up of individuals with radiologically isolated syndrome, suggesting the presence of a presymptomatic phase in patients with PPMS [25]. Among the $34 \%$ of patients with radiologically isolated syndrome who developed an initial clinical event 
related to demyelination within the CNS over a 5-year follow-up period, $11.7 \%$ fulfilled the criteria for PPMS [25].

\section{Previous Trials}

Nearly 50 phase II or III clinical trials including patients with progressive forms of MS have been completed over the last 30 years [18]. The major strata of therapeutics studied included immunosuppressants or chemotherapeutics, immunomodulators, and neuroprotectants [18]. The only disease-modifying drug specifically approved by the FDA for SPMS is mitoxantrone. However, the use of this agent is limited because of its cardiotoxic effects and lifetime risk of leukemia postexposure [26]. Currently, there are no FDA-approved therapies specific for the treatment of PPMS [2].

Therapeutics that failed to show "clinically significant and reproducible efficacy" in reducing or halting disability progression in progressive MS, based on phase III trials, included azathioprine [27], ciclosporin [28], cyclophosphamide [29], plasma exchange [29], intravenous (IV) immunoglobulin [30] (though 1 study showed a borderline significant delay in time to sustained progression on Expanded Disability Status Scale (EDSS) in patients with PPMS) [31], sulfasalazine [32], interferon (IFN)- $\beta 1$ a [33] and IFN- $\beta 1 \mathrm{~b}[34,35]$, glatiramer acetate [36], linomide (study terminated early owing to serious cardiopulmonary side effects and pancreatitis) [37], cladribine [38], rituximab (IV infusion) [14], MBP8298 (dirucotide) [39], dronabinol [40], hydroxyurea (no efficacy based on interim analysis) [41], alemtuzumab (phase II) [42], and, most recently, fingolimod [43]. While unsuccessful trials of immunomodulating and immunosuppressant medications propose a shift to neuroprotective and neuroregenerative strategies, it is important to distinguish between failed trials and failed therapeutics [18, 44]. For instance, as we will point out later, although a trial of IV rituximab in patients with PPMS failed to show any significant beneficial impact on disease progression [14], subgroup analyses suggested a possible benefit in younger patients ( $\leq 51$ years of age) with inflammatory lesions on magnetic resonance imaging (MRI). This has encouraged a trial of combined IV and intrathecal rituximab given the possibility of intrathecal compartmentalization of inflammatory cells. Another example applies to the failed trial of fingolimod for PPMS in which $>40 \%$ of patients were $>50$ years of age, and $>85 \%$ had no gadolinium-enhancing lesions on MRI at baseline [43]. Taking into account the result of subgroup analysis from the rituximab study, a subgroup analysis of the failed fingolimod trial might be informative.

\section{Therapeutics Under Investigation}

Here, we review the various therapies under study for progressive forms of MS, in alphabetical order.

\section{Adrenocorticotropic hormone}

While adrenocorticotropic hormone (ACTH) has been approved for use in MS exacerbations for many years because of its steroidogenic properties, recent data suggest that ACTH may benefit certain conditions through corticosteroid-independent mechanisms [45]. For instance, ACTH has been shown to improve brain function in infantile spasms (an epileptic encephalopathy of early infancy with limited treatment options) compared with the use of glucocorticosteroids $[45,46]$. It has been suggested that ACTH exerts anti-inflammatory effects (via modulation of regulatory $\mathrm{T}$ cells, inhibition of activation of nuclear factor kappa $\mathrm{B}$ (NF- $\mathrm{kB}$ ), and possibly triggering CNS-restricted release of noradrenalin and acetylcholine), and may have neuroprotective effects in spinal cord injury and ischemic brain injury [45, 47]. These aspects provide support for future scientific efforts in progressive MS. A phase II trial of ACTH in progressive MS is underway (NCT01950234).

\section{Biotin}

Biotin is a water-soluble vitamin belonging to the $\mathrm{B}$ complex family, and an essential micronutrient that acts as a cofactor for decarboxylase enzymes [48]. High doses of biotin have been found to prevent the progression and even reverse the course of "biotin-responsive basal ganglia disease", an autosomal recessive disorder characterized by subacute encephalopathy of childhood onset with extrapyramidal and pyramidal manifestations [49, 50]. Recently, a small case series of patients with a characteristic leukodystrophy involving periventricular white matter, corticospinal tract, cerebellar peduncles, and optic radiations, with clinical manifestations of relapsing episodes of cerebellar ataxia and optic neuropathy were reported to respond to high doses of biotin [51]. One of those patients was later discovered to have SPMS [52]. In an open-label pilot study, high doses of biotin (100-300 mg/day) in patients with SPMS and PPMS were shown to have an impact on disease progression in those with optic neuropathy, homonymous hemianopia, and spinal cord involvement [52]. The preliminary results of a phase III trial of high-dose biotin (MD1003) in progressive MS (NCT02220933) has shown improvement in MS-related disability outcomes (13\% of the exposed group vs $0 \%$ of placebo group had improvement of Expanded Disability Status Scale or timed 25-foot walk at month 9 (confirmed at month 12) [53].

\section{Amiloride, Fluoxetine, and Riluzole}

Amiloride is a potassium-sparing diuretic capable of inhibiting acid-sensing ion channels, a property that has been linked to possible neuroprotective effects [54, 55]. It has been found to reduce functional neurologic deficits in experimental allergic encephalomyelitis (EAE) studies [54, 55]. Riluzole, is 
an inhibitor of tetradotoxin-sensitive voltage-gated sodium channels, with antiglutamatergic profile, and the only established disease-modifying treatment for amyotrophic lateral sclerosis [56]. Fluoxetine is a selective serotonin reuptake inhibitor that has been suggested to have neuroprotective properties by supression of microglia activation, and NF- $\mathrm{kB}$ (a family of transcription factors with an essential role in inflammation and innate immunity) activity, and enhancement of the production of the brain-derived neurotrophic factor in animal models [57-59]. A 4-arm phase II trial of amiloride, fluoxetine, and riluzole (compared with placebo) is currently in progress (NCT01910259).

\section{Anti-leucine-rich Repeat and Immunoglobulin-like Domain Containing Neurite Outgrowth Inhibitor Receptor-interacting Protein-1 Antibody}

Leucine-rich repeat and immunoglobulin-like domain containing neurite outgrowth inhibitor receptor-interacting protein-1 (LINGO-1) is a cell surface protein expressed in neural cells, and a negative modulator of axonal myelination via inhibition of the differentiation of oligodendrocyte precursor cells to mature oligodendrocytes [60,61]. Blockage of LINGO-1 may therefore represent a potential strategy for remyelination, and axonal preservation in MS [62]. In an EAE study, anti-LINGO-1 antibodies improved axonal integrity and new myelin sheath formation, resulting in functional recovery. In a recent phase II trial of BIIB033 (a novel anti-LINGO-1 monoclonal antibody) in patients with a first acute optic neuritis episode, results showed an improved average difference in latency recovery $(7.55 \mathrm{~ms}$ at 24 weeks; $p=$ 0.05 ) as measured by full-field visual evoked potential) [63]. A separate phase II trial of BIIB033 to investigate the impact of anti-LINGO-1 on disease progression in patients with RRMS and SPMS is currently ongoing (NCT01864148).

\section{Domperidone}

It is well known that dopamine plays a key role in the regulation of prolactin secretion mainly by inhibiting the anterior pituitary lactotrophs [64]. Domperidone is a dopamine-2 receptor antagonist widely used in Canada and Europe as a prokinetic agent for gastroparesis, and to induce lactation by increasing prolactin secretion (as a side effect) [65]. EAE studies suggest that prolactin can promote myelin repair [66, 67], and thereby may have a potential role as a remyelinating therapy in MS $[62,66]$. These data are in alignment with the well-established data of the benefits of pregnancy in reducing disease activity in MS, an observation that may be, in part, related to higher prolactin levels [68]. A phase II trial of oral domperidone in patients with SPMS is currently underway in Canada (NCT02308137).

\section{Erythropoietin}

Increasing evidence suggest that erythropoietin (EPO), originally known as hematopoietic growth factor, is part of a potent endogenous neuroprotective system in the brain [69]. EPO has been shown to have antiapoptotic, antioxidative, anti-inflammatory, neurotrophic, and plasticity-modulating effects in animal models of various neurological diseases [70]. In EAE studies, EPO has been shown to reduce clinical severity, axonal injury and demyelination, and diminish glial expression of major histocompatibility complex class II [71]. A small-sized open-label pilot study of EPO in patients with progressive MS showed both clinical and electrophysiological improvement of motor function with high-dose EPO [72]. A phase II trial of EPO in progressive MS is planned (NCT01144117).

\section{Hematopoetic Stem Cell Therapy}

Hematopoietic stem cell transplantation has been proposed as a second-line therapy for refractory MS, presumably by eradicating the autoreactive cells, resetting the aberrant immune response to self-antigens, and possibly promoting regenerative processes in the CNS [73, 74]. Early clinical trials showed only a modest benefit $[75,76]$; however, long-term follow-up results suggested a significant improvement in disease progression-free survival rate in patients with active inflammation [77]. Over the last decade, multiple efforts have been made to optimize the cell source, patient selection, and the safety and tolerability of stem cell-based therapies [74]. In a recent phase II trial, high-dose immunosuppressive therapy and autologous hematopoietic cell transplantation without maintenance therapy was found to be effective for inducing sustained remission of active RRMS at 3 years [78]. While autologous hematopoietic stem cell transplantation is based on utilizing hematopoietic cells to repopulate the immune system following high-dose immunosuppression, infusion of mesenchymal cells or autologous unfractionated bone marrow is not preceded by immunosuppression. In an open-label proof-of-concept trial in patients with SPMS with clinical evidence of optic nerve involvement, infusion of autologous bone marrow-derived mesenchymal stem cells improved visual acuity and visual evoked response latency, and increased optic nerve area [79]. A phase II trial of autologous bone marrow infusion in patients with SPMS or PPMS is in progress (NCT01815632).

\section{Ibudilast}

Ibudilast is a nonselective phosphodiesterase inhibitor that has been suggested to have immunomodulatory and neuroprotective effects $[80,81]$. Ibudilast inhibits leukotrienes and nitric oxide synthesis, and reduces tumor necrosis factor- $\alpha$ from astrocytes and microglial cells $[81,82]$. A phase II trial of 
ibudilast in patients with RRMS demonstrated no beneficial effect on the rate of newly active lesions and relapses but reduced brain atrophy rate, suggesting a potential neuroprotective role [83]. A phase II trial of ibudilast in progressive MS is in progress (NCT01982942).

\section{Idebenone}

Idebenone, is a synthetic analog of coenzyme Q10, an endogenous antioxidant found in all cellular membranes, and a constituent of the adenosine triphosphate-producing electron transport chain of mitochondria [84]. In addition to antioxidant properties, idebenone has been shown to exert anti-inflammatory effects in vitro [85]. Idebenone use in Friedreich's ataxia and Leber's hereditary optic neuropathy, both of which are thought to be mitochondrial disorders, has shown beneficial effects. In a recent animal study, idebenone was found to protect hippocampal HT22 cells from glutamate-induced cell death in vitro, although idebenone-treated EAE mice did not exhibit any clinical benefit with respect to reducing inflammation, demyelination, and axonal injury [86]. Given that mitochondrial dysfunction may play a key role in progressive axonal loss in MS, further investigations as a therapeutic option for progressive forms of MS are rational. A phase II trial of idebenone in PPMS is currently underway (NCT01854359).

\section{Lipoic Acid}

Lipoic acid is a natural antioxidant with signal transduction modulatory pathways, and has been suggested as a potential therapeutic agent in diseases associated with oxidative stress such as diabetic neuropathy, Alzheimer's disease, and MS [87]. Lipoic acid has been shown to suppress EAE by inhibiting the entry of T cells into the CNS [88]. In a recent small size phase I study, lipoic acid was well tolerated in patients with MS and associated with a reduction in matrix metalloproteinase-9 and soluble intercellular adhesion molecule-1 [89]. A phase II/III trial of lipoic acid in patients with SPMS is underway (NCT01188811).

\section{Lithium}

Accumulating evidence indicates that lithium, one of the oldest antipsychotic medications, exhibits its therapeutic effects through inhibition of glycogen synthase kinase-3 (GSK-3), a serine-threonine protein kinase and major regulator of inflammation [90]. It has been shown in EAE studies that pre-treatment with lithium suppressed the onset of disease activity [91]. When lithium was administered after the induction of EAE, it reduced disease severity and facilitated partial recovery [91]. It has therefore been suggested that GSK-3 could be a target for future treatments in MS. A pilot phase
I/II trial of lithium in progressive forms of MS is underway (NCT01259388).

\section{Masitinib}

Masitinib is a selective tyrosine kinase inhibitor that modulates migration, survival, and degranulation of mast cells [92]. Increasing evidence suggests that mast cells actively play a role in pathogenesis of MS by releasing vasoactive mediators that sustain inflammatory cascade, disrupting the BBB, and stimulating activated $\mathrm{T}$ cells, amongst other mechanisms [93, 94]. In a phase IIa proof-of-concept trial, masitinib was, overall, well tolerated and found to have positive but not statistically significant effect on clinical progression in patients with progressive MS [95]. A phase IIb/III study of masitinib in patients with relapse-free SPMS or PPMS is in progress (NCT01433497).

\section{MIS416}

MIS416 is a myeloid-directed microparticle immune response modifier (derived from Propionibacterium acnes), which was originally developed as a vaccine adjuvant [96]. MIS416 has been suggested to modulate T-cell-mediated autoimmune responses in EAE by simultaneously activating innate Toll-like receptor 9 and nucleotide-binding oligomerization domain-containing protein 2 [96]. The restricted uptake of MIS416 by phagocytic cells has been suggested to lead to targeted modulation of the innate immune system [97]. MIS416 was initially used in patients with SPMS outside of a formal clinical trial setting under compassionate use legislation in New Zealand. Recently, in a phase Ib/IIa clinical trial, MIS416 was shown to suppress the development of proinflammatory $\mathrm{T}$ helper (Th)1, Th2, and Th17 cells in EAE, and to increase the serum levels of IFN- $\gamma$ and IFN- $\gamma$-associated proteins in 19 patients with SPMS [97]. A phase IIb trial is underway to further investigate the effect of MIS416 in progressive forms of MS (NCT02228213) [98].

\section{Natalizumab}

Monoclonal antibodies, first used to treat cancer, are highly specific immunoglobulins against candidate target antigens, the discovery of which has revolutionized the treatment of autoimmune diseases [99]. Two monoclonal antibodies have been approved for treatment of relapsing forms of MS [natalizumab (Tysabri; Biogen, Research Triangle Park, NC, USA) and alemtuzumab (Lemtrada; Genzyme, Cambridge, MA, USA)], with several more are under investigations in clinical trials or preclinical development phase.

Natalizumab is a humanized IgG4 monoclonal antibody that targets the $\alpha 4$-chain of $\alpha 4 \beta 1$ integrin and other $\alpha 4$-integrin-containing adhesion molecules, thereby 
inhibiting the adhesion of lymphocytes to the endothelium and blocking lymphocyte extravasation into the CNS through the BBB [100]. In an open-label phase IIa proof-of-concept trial of IV natalizumab in patients with progressive MS, results showed reductions in CSF inflammatory biomarkers, specifically osteopontin, chemokine (C-X-C motif) ligand 13 and matrix metalloproteinase-9, as well as CSF markers of axonal damage and demyelination [i.e., neurofilament light chain, and myelin basic protein (MBP)], thereby supporting potential beneficial effect of natalizumab in progressive MS [101]. Preliminary results from a phase III trial of natalizumab in patients with SPMS (ASCEND; NCT01416181) showed that the study did not achieve its composite primary end point, defined as the percentage of patients with confirmed progression of disability on one or more of the following components: EDSS, Timed 25-Foot Walk, and 9-Hole Peg Test [102].

\section{NeuroVax}

Selective immunotherapies targeted to restore self-tolerance to the autoantigens presumed responsible for the pathogenesis of MS remains an interesting approach. As such, several vaccine strategies including T cell, T-cell receptor (TCR) peptide, and DNA-based vaccinations have been pursued and, on the surface, appear to reflect more selective therapies for MS [103-104]. In an open-label single arm study of 27 patients with MS (including 10 patients with SPMS and 3 patients with PPMS), monthly intramuscular injection of a trivalent TCR peptide induced a surge of proliferating interleukin-1-secreting TCR peptide-specific $\mathrm{T}$ cells concomitant with restoration of deficient expression of forkhead box P3 (an important transcription factor in the development and function of regulatory T cells) to higher than normal levels $[105,106]$. A phase II study of NeuroVax (a TCR peptide vaccine; Immune Response BioPharma, Atlantic City, NJ, USA) in patients with SPMS is planned to be started soon (NCT02057159).

\section{Phenytoin, and Oxcarbazepine}

It has been shown in experimental animal models that loading of partially demyelinated axons with sodium ions results in an accumulation of calcium ions, which triggers a cascade of degradative enzyme activity and finally leads to axonal degeneration [107]. Partial blockade of sodium channels is therefore thought to have neuroprotective properties [107-109]. As such, sodium-blocking agents such as phenytoin, lamotrigine, carbamazepine, or oxcarbazepine, and even newer agents such as safinamide and flecainide have been suggested to have potential neuroprotective effects in MS [109].

In a phase II trial of lamotrigine versus placebo in patients with SPMS, lamotrigine treatment was unexpectedly found to be associated with greater annual cerebral volume loss in the first year compared with placebo; an effect reversed partially on discontinuation of treatment [110]. However, the rate of decline on Timed 25-Foot Walk speed assessments was reduced in the treatment group compared with placebo [110]. The interpretation of the results was also further complicated by a nonadherence rate of up to $50 \%$ in lamotrigine group.

It is known that treatment of acute optic neuritis, a common manifestation of MS, with steroids has little or no impact on the eventual extent of recovery [111]. Optical coherence tomography of retinal nerve fiber layer and MRI of optic nerve following optic neuritis have shown volume loss (neuroaxonal loss) in correlation with impaired visual function [112]. In a phase II trial of phenytoin versus placebo in patients with acute optic neuritis, treatment with phenytoin $(4 \mathrm{mg} / \mathrm{kg} /$ day $)$ within 2 weeks of symptom onset was found to have a $30 \%$ protective effect on retinal nerve fiber layer thickness and a $34 \%$ protective effect on macular volume at 6 months following acute optic neuritis, using optical coherence tomography [113].

A phase II trial of oxcarbazepine in SPMS assessing the change in the content of neurofilament light chain in CSF, a proposed surrogate marker of neurodegeneration, as well as clinical disability and imaging outcomes is underway (NCT02104661).

\section{Rituximab and Ocrelizumab: 2 Anti-CD20 Monoclonal Antibodies}

Rituximab is a chimeric IgG1 monoclonal antibody that targets CD20 on B cells, thereby depleting B cells by several mechanisms, including apoptosis, direct antibody-dependent cellular cytotoxicity, and complement-dependent cytolysis [100]. Although rituximab is not FDA-approved for MS, it is commonly used as an off-label therapy. A phase III trial of IV rituximab in patients with PPMS did not show any significant difference in time to reach confirmed disease progression. The results from preplanned subgroup analyses, however, suggested that it may affect disease progression in younger patients, particularly those with inflammatory lesions on brain MRI [14]. Given that the progressive phase of MS is thought to be associated with intrathecal compartmentalization of inflammatory cells, it has been suggested that intrathecal administration of immunosuppressants may represent a promising new approach in progressive MS [114]. A phase II trial of combined IV and intrathecally administered rituximab ( $v s$ placebo) in patients with SPMS is currently ongoing (NCT01212094). This is also the first trial to monitor immune activity in the CNS of patients with MS using a microdialysis catheter for CSF sampling [8].

Ocrelizumab is another IgG1 monoclonal antibody against CD20 B cells. In contrast to rituximab, it is a humanized monoclonal antibody, thereby having fewer anti-idiotypic reactions [100]. Prompted by positive results from the subgroup analysis of the IV rituximab trial [14], a phase III trial of ocrelizumab in patients with PPMS with the inclusion criteria adjusted based on the subgroup analysis was designed (NCT01194570). 
Preliminary results from the ORATORIO study showed that patients who were treated with ocrelizumab had a $24 \%$ lower risk of confirmed disability progression after 12 weeks than those who were treated with the placebo [115].

\section{Simvastatin}

Statins (hydroxymethylglutaryl-CoA reductase inhibitors) are widely prescribed and well tolerated in the treatment of hypercholesterolemia. However, they also exert immunomodulatory and neuroprotective properties and have been shown to improve cerebrovascular hemodynamics [116]. These properties make statins an attractive candidate drug in patients with later stages of MS when dysfunction of brain parenchymal cells and vascular endothelial cells occur $[117,118]$. Studies of statins in EAE and open-label trials in patients with MS have shown decreased disease activity $[119,120]$. In a recent phase II trial of simvastatin versus placebo in patients with SPMS, simvastatin was found to reduce significantly the annualized rate of whole brain atrophy compared with placebo $[-0.25 \%$ per year adjusted difference in atrophy rate between the 2 groups ( $p=0.003$ ), a $43 \%$ reduction in annualized rate of atrophy] [121]. These results provide support for the advancement to a phase III trial of statins for progressive MS.

\section{Siponimod}

Siponimod (BAF312) is an oral modulator of sphingosine 1-phosphate (S1P) receptor that acts selectively on S1P-1 and S1P-5, demonstrating greater selectivity than fingolimod [122]. It reduces recirculation and infiltration of potentially autoaggressive lymphocytes into the CNS and may have direct CNS effects by modulating neurobiological processes via S1P-1 and S1P-5 receptors on astrocytes and oligodendrocytes [123, 124]. S1P receptor modulators have been suggested to exert neuroprotective and regenerative actions in the CNS, in addition to anti-inflammatory effects [125]. In an adaptive dose-ranging phase II trial in patients with RRMS, siponimod was shown to reduce brain MRI lesions and relapses by up to $80 \%$ (vs placebo) [126]. A phase III trial of siponimod in patients with SPMS is currently in underway (NCT01665144) [127].

\section{Sunphenon Epigallocatechin-3-gallate}

Sunphenon epigallocatechin-3-gallate (EGCg), a major constituent of green tea, has emerged as a potential neuroprotective agent for the treatment of neurological disorders associated with harmful effects of reactive oxygen species [128, 129]. In EAE studies, EGCg has been shown to have both anti-inflammatory properties by influencing T-cell proliferation and inhibiting the activation of $\mathrm{NF}-\mathrm{kB}$, and neuroprotective properties by acting as a free radical scavenger $[130,131]$. A phase II/III trial of oral Sunphenon
(Taiyo International, Minneapolis, MN, USA) EGCg in patients with progressive MS is underway (NCT00799890).

\section{Tcelna}

Autologous T-cell immunotherapy has been suggested to deplete or regulate the pathogenic myelin reactive $\mathrm{T}$ cells that maintain autoimmune processes within the CNS of patients with MS [132, 133]. Tcelna (imilecleucel-T; Opexa Therapeutics, The Woodlands, TX, USA), formerly known as Tovaxin, is a T-cell immunotherapy that consists of an autologous pool of T-cell lines raised against selected immunodominant peptides derived from MBP, myelin oligodendrocyte glycoprotein (MOG), and proteolipid protein [134]. The immunodominant peptides are selected on an individual basis by prescreening for reactivity against overlapping peptide libraries including the above-mentioned 3 major myelin antigens [134]. Tcelna is therefore suggested to be specifically tailored to each patient's immune response profile to putative myelin autoantigens. A phase II trial of subcutaneous injection of Tcelna in patients with SPMS is underway (NCT01684761).

\section{Future Research Directions, and Conclusions}

The long list of therapeutic agents currently in development for the treatment of PPMS and SPMS provides some reason for optimism that molecular and cellular therapeutic targets will eventually be identified that modulate these 2 clinical phenotypes.

Previous failed clinical trials illustrate that broad targeting of adaptive immune responses outside the CNS against currently unidentified autoantigens is not a viable strategy, and that inflammatory activity likely shifts towards innate immune responses within the brain and spinal cord in later disease stages. In addition, accumulation of myelin loss, axonal injury, and subsequent neurodegeneration amplify the clinical impact of this chronic disorder.

Neurologists, clinical neuroscientists, and neuroimmunologists have long struggled to develop beneficial pharmacotherapies for the progressive phase of MS. The reasons are multiple and include 1) an incomplete understanding of the disease process; 2) the lack of animal models that reliably predict therapeutic responses in patients; and 3) agents that have good CNS bioavailability. Also, clinical trial designs that have been employed in patients with RRMS may be less relevant to progressive forms of MS.

The EAE model is still employed to identify potentially effective pharmacological agents for MS. In experimental mice, depending on the encephalitogenic antigen and the major histocompatibility complex genotype, different clinical EAE disease courses are observed [135]. When induced in $\mathrm{PL} / \mathrm{J}$ and B10.PL $(\mathrm{H}-2 \mathrm{u})$ mice, EAE is typically acute and 
monophasic. In SJL (H-2s) mice, the disease course is relapsing-remitting. Vaccination of C57BL/6 (H-2b) mice MOG peptide 35-55 results in a chronic progressive disease course, resembling PPMS. Levy et al. [136] recently demonstrated that immunization of non-obese diabetic mice with $\mathrm{MOG}_{\mathrm{p} 35-55}$ initially leads to relapsing-remitting EAE between days 20-70 after inoculation, followed by a chronic progressive stage. This experimental model is currently considered by many to be the most relevant in reflecting the initiation of RRMS and subsequent transitioning to SPMS. While glatiramer acetate and natalizumab were tested in EAE and subsequently successfully developed in clinical trial programs, a large number of therapeutic agents that ameliorated EAE failed in patients with MS.

Ideally, clinical trials for progressive MS should not only test a potential therapeutic, but also develop meaningful clinical trial methodology. Ontaneda et al. [18], in a recent review article, eloquently formulated several recommendations for key aspects of future trials of progressive MS (intervention selection, study participants, trial design, outcome measures, and CSF biomarkers) based on lessons learned from prior clinical trials. Further research on the identification of clinically meaningful outcomes for MS clinical trials should be encouraged. Given prior instances of early termination of unsuccessful clinical trials in progressive MS, and the importance of efficient completion of trials, application of novel trial designs should be considered [137]. An adaptive design helps to address uncertainties about choices made during the planning phase of a trial by allowing modifications to trial and/or statistical methods of ongoing trials [138]. Heterogeneity of findings should be assessed based on predefined subgroup analysis, and not post hoc exploratory analysis. Multi-arm trials should to be encouraged as investigation of multiple medications simultaneously minimizes exposure time to placebo and cost, and can potentially result in faster ways of finding effective treatments [139]. Drug repurposing needs to be considered; yet, developing systemic and rational approaches for the identification of repurposing candidates in order to maximize the chance of success in clinical development remains a challenge [140]. Although fully validated and reliable biomarkers still remains to be identified, even in relapsing-remitting MS, the challenge is when it comes to progressive forms of MS given that many of the studied biomarkers so far are aimed at capturing the predominantly inflammatory disease activity rather than the neurodegenerative aspects of the disease. While this continues to remain an active area of research, overall it appears that brain atrophy is the preferred method of monitoring the neurodegenerative process in progressive MS [141]. In addition, axonal- and glial-based biomarkers in CSF (e.g., neurofilament chains [142, 143], glial fibrillary acidic protein, tau and S100b protein) [144], and metabolomics profiling methods have received much attention [145]. CSF analysis and banking, and biomarker adaptive designs have therefore been encouraged in trials of progressive MS [146]. Last, but not least, global research collaboration is also a necessity to overcome the challenges of progressive MS, as evidenced by multiple lines of research boosted by the Progressive MS Alliance over the last few of years.

Overall, given ongoing and planned clinical trial initiatives, and the largest ever focus of the research community on progressive MS, future prospects for developing targeted disease-modifying drugs for progressive forms of MS appear promising.

\section{Compliance with Ethical Standards}

Required Author Forms Disclosure forms provided by the authors are available with the online version of this article.

Disclosures A.S. received a postdoctoral fellowship award from the Multiple Sclerosis (MS) Society of Canada, and was funded by grants from the National MS Society and the Canadian Institutes of Health Research. A.S. has received travel grants from the European Committee for Treatment and Research in Multiple Sclerosis, the endMS Research and Training Network, and the Consortium of MS Centers. D.O. has received lecture fees from Acorda Therapeutics, Genzyme, and TEVA Neuroscience, consulting and advisory board fees from Genzyme and TEVA Neuroscience, and research support from Biogen. O.S. serves on the editorial boards of JAMA Neurology, Multiple Sclerosis Journal, and Therapeutic Advances in Neurological Disorders. O.S. has received grant support from Teva Pharmaceuticals and Opexa Therapeutics. O.S. has served on data monitoring committees for Pfizer and Sanofi-Aventis without monetary compensation. O.S. has served on an advisory board for Sanofi Genzyme and Genentech, and has advised Huron Life Sciences. O.S. is funded by a Merit grant from the US Department of Veterans Affairs.

\section{References}

1. Compston A, Coles A. Multiple sclerosis. Lancet 2008;372:15021517.

2. Ransohoff RM, Hafler DA, Lucchinetti CF. Multiple sclerosis-a quiet revolution. Nat Rev Neurol 2015;11:134-142.

3. National MS Society. The MS disease modifyinf medicatiobs. Available at: http://www.nationalmssociety.org/NationalMSSociety/ media/MSNationalFiles/Brochures/Brochure-The-MS-DiseaseModifying-Medications.pdf. Accessed June 7, 2015.

4. Feinstein A, Freeman J, Lo AC. Treatment of progressive multiple sclerosis: what works, what does not, and what is needed. Lancet Neurol 2015;14:194-207.

5. Ontaneda D, Fox RJ. Progressive multiple sclerosis. Curr Opin Neurol 2015;28:237-243.

6. Browne P, Chandraratna D, Angood C, et al. Atlas of multiple sclerosis 2013: a growing global problem with widespread inequity. Neurology 2014;83:1022-1024.

7. Thompson AJ. A much-needed focus on progression in multiple sclerosis. Lancet Neurol 2015;14:133-135.

8. International Progressive MS Alliance. Facts and figures. Available at: http://www.progressivemsalliance.org/progressivems/facts-and-figures/. Accessed June 4, 2015.

9. Fox RJ, Thompson A, Baker D, et al. Setting a research agenda for progressive multiple sclerosis: the International Collaborative on Progressive MS. Mult Scler 2012;18:1534-1540. 
10. Mahad DH, Trapp BD, Lassmann H. Pathological mechanisms in progressive multiple sclerosis. Lancet Neurol 2015;14:183-193.

11. Fischer MT, Wimmer I, Hoftberger R, et al. Disease-specific molecular events in cortical multiple sclerosis lesions. Brain 2013;136:1799-1815.

12. Davies AL, Desai RA, Bloomfield PS, et al. Neurological deficits caused by tissue hypoxia in neuroinflammatory disease. Ann Neurol 2013;74:815-825.

13. Hametner S, Wimmer I, Haider L, Pfeifenbring S, Bruck W, Lassmann $\mathrm{H}$. Iron and neurodegeneration in the multiple sclerosis brain. Ann Neurol 2013;74:848-861.

14. Hawker K, O'Connor P, Freedman MS, et al. Rituximab in patients with primary progressive multiple sclerosis: results of a randomized double-blind placebo-controlled multicenter trial. Ann Neurol 2009;66:460-471.

15. Magliozzi R, Howell O, Vora A, et al. Meningeal B-cell follicles in secondary progressive multiple sclerosis associate with early onset of disease and severe cortical pathology. Brain 2007;130:10891104.

16. Choi SR, Howell OW, Carassiti D, et al. Meningeal inflammation plays a role in the pathology of primary progressive multiple sclerosis. Brain 2012;135:2925-2937.

17. Kowarik MC, Pellkofer HL, Cepok S, et al. Differential effects of fingolimod (FTY720) on immune cells in the CSF and blood of patients with MS. Neurology 2011;76:1214-1221.

18. Ontaneda D, Fox RJ, Chataway J. Clinical trials in progressive multiple sclerosis: lessons learned and future perspectives. Lancet Neurol 2015;14:208-223.

19. Brinar VV, Barun B. Challenges in multiple sclerosis; how to define occurence of progression. Clin Neurol Neurosurg 2013;115(Suppl. 1):S30-S34.

20. Lublin FD, Reingold SC, Cohen JA, et al. Defining the clinical course of multiple sclerosis: the 2013 revisions. Neurology 2014;83:278-286.

21. Lassmann H, Bruck W, Lucchinetti CF. The immunopathology of multiple sclerosis: an overview. Brain Pathol 2007;17:210-218.

22. Scalfari A, Neuhaus A, Daumer M, Ebers GC, Muraro PA. Age and disability accumulation in multiple sclerosis. Neurology 2011;77:1246-1252.

23. Confavreux C, Vukusic S. Natural history of multiple sclerosis: a unifying concept. Brain 2006;129:606-616.

24. Paz Soldan MM, Novotna M, Abou Zeid N, et al. Relapses and disability accumulation in progressive multiple sclerosis. Neurology 2015;84:81-88

25. Kantarci OH, Lebrun C, Siva A, et al. Primary progressive MS evolving from radiologically isolated syndrome. Ann Neurol 2015. doi:10.1002/ana.24564.

26. Okuda DT. Immunosuppressive treatments in multiple sclerosis. Handb Clin Neurol 2014;122:503-511.

27. Anon. Double-masked trial of azathioprine in multiple sclerosis. British and Dutch Multiple Sclerosis Azathioprine Trial Group. Lancet 1988;2:179-183.

28. Anon. Efficacy and toxicity of cyclosporine in chronic progressive multiple sclerosis: a randomized, double-blinded, placebocontrolled clinical trial. The Multiple Sclerosis Study Group. Ann Neurol 1990;27:591-605.

29. Anon. The Canadian cooperative trial of cyclophosphamide and plasma exchange in progressive multiple sclerosis. The Canadian Cooperative Multiple Sclerosis Study Group. Lancet 1991;337: 441-446.

30. Hommes OR, Sorensen PS, Fazekas F, et al. Intravenous immunoglobulin in secondary progressive multiple sclerosis: randomised placebo-controlled trial. Lancet 2004;364:11491156

31. Pohlau D, Przuntek H, Sailer M, et al. Intravenous immunoglobulin in primary and secondary chronic progressive multiple sclerosis: a randomized placebo controlled multicentre study. Mult Scler 2007;13:1107-1117.

32. Noseworthy JH, O'Brien P, Erickson BJ, et al. The Mayo ClinicCanadian Cooperative trial of sulfasalazine in active multiple sclerosis. Neurology 1998;51:1342-1352.

33. Andersen O, Elovaara I, Farkkila M, et al. Multicentre, randomised, double blind, placebo controlled, phase III study of weekly, low dose, subcutaneous interferon beta-1a in secondary progressive multiple sclerosis. J Neurol Neurosurg Psychiatry 2004;75:706-710.

34. Panitch H, Miller A, Paty D, Weinshenker B, North American Study Group on Interferon beta-1b in Secondary Progressive MS. Interferon beta- $1 b$ in secondary progressive MS: results from a 3-year controlled study. Neurology 2004;63:1788-1795.

35. Anon. Placebo-controlled multicentre randomised trial of interferon beta- $1 \mathrm{~b}$ in treatment of secondary progressive multiple sclerosis. European Study Group on interferon beta-1b in secondary progressive MS. Lancet 1998;352:1491-1497.

36. Wolinsky JS, Narayana PA, O'Connor P, et al. Glatiramer acetate in primary progressive multiple sclerosis: results of a multinational, multicenter, double-blind, placebo-controlled trial. Ann Neurol 2007;61:14-24.

37. Noseworthy JH, Wolinsky JS, Lublin FD, et al. Linomide in relapsing and secondary progressive MS: part I: trial design and clinical results. North American Linomide Investigators. Neurology 2000;54:1726-1733.

38. Rice GP, Filippi M, Comi G. Cladribine and progressive MS: clinical and MRI outcomes of a multicenter controlled trial. Cladribine MRI Study Group. Neurology 2000;54:1145-1155.

39. Freedman MS, Bar-Or A, Oger J, et al. A phase III study evaluating the efficacy and safety of MBP8298 in secondary progressive MS. Neurology 2011;77:1551-1560.

40. Zajicek J, Ball S, Wright D, et al. Effect of dronabinol on progression in progressive multiple sclerosis (CUPID): a randomised, placebo-controlled trial. Lancet Neurol 2013;12:857-865.

41. Bot A, Smith D, Bot $\mathrm{S}$, et al. Plasmid vaccination with insulin $\mathrm{b}$ chain prevents autoimmune diabetes in nonobese diabetic mice. $\mathrm{J}$ Immunol 2001;167:2950-2955.

42. Coles AJ, Cox A, Le Page E, et al. The window of therapeutic opportunity in multiple sclerosis: evidence from monoclonal antibody therapy. J Neurol 2006;253:98-108.

43. Lublin FD, Miller D, Freedman M, et al. Oral fingolimod versus placebo in patients with primary progressive multiple sclerosis (PPMS): results of the INFORMS phase III trial. . The 67th Annual Meeting of the American Academy of Neurology, Washington, DC, April 18-25, 2015.

44. Wiendl H, Hohlfeld R. Therapeutic approaches in multiple sclerosis: lessons from failed and interrupted treatment trials. BioDrugs 2002;16:183-200.

45. Arnason BG, Berkovich R, Catania A, Lisak RP, Zaidi M. Mechanisms of action of adrenocorticotropic hormone and other melanocortins relevant to the clinical management of patients with multiple sclerosis. Mult Scler 2013;19:130-136.

46. Stafstrom CE, Arnason BG, Baram TZ, et al. Treatment of infantile spasms: emerging insights from clinical and basic science perspectives. J Child Neurol 2011;26:1411-1421.

47. Catania A. Neuroprotective actions of melanocortins: a therapeutic opportunity. Trends Neurosci 2008;31:353-360.

48. Said HM. Biotin: the forgotten vitamin. Am J Clin Nutr 2002;75: 179-180.

49. Ozand PT, Gascon GG, Al Essa M, et al. Biotin-responsive basal ganglia disease: a novel entity. Brain 1998;121:1267-1279.

50. Tabarki B, Al-Shafi S, Al-Shahwan S, et al. Biotin-responsive basal ganglia disease revisited: clinical, radiologic, and genetic findings. Neurology 2013;80:261-267. 
51. Sedel F, Challe G, Vignal C, Assouad R, Bellanger A, Galanaud D. A novel biotin-sensitive leukodystrophy (BSL). J Inherit Metab Dis 2011;34:S267.

52. Sedel F, Papeix C, Bellanger A, et al. High doses of biotin in chronic progressive multiple sclerosis: a pilot study. Mult Scler Relat Disord 2015;4:159-169.

53. Tourbah A, Frenay CL, Edan G, et al. Effect of MD1003 (high doses of biotin) in progressive multiple sclerosis: results of a pivotal phase III randomized double blind placebo controlled study. The 67th Annual Meeting of the American Academy of Neurology, Wasgington, DC, April 18-25, 2015.

54. Vergo S, Craner MJ, Etzensperger R, et al. Acid-sensing ion channel 1 is involved in both axonal injury and demyelination in multiple sclerosis and its animal model. Brain 2011;134:571-584.

55. Friese MA, Craner MJ, Etzensperger R, et al. Acid-sensing ion channel-1 contributes to axonal degeneration in autoimmune inflammation of the central nervous system. Nat Med 2007;13: 1483-1489.

56. Cheah BC, Vucic S, Krishnan AV, Kiernan MC. Riluzole, neuroprotection and amyotrophic lateral sclerosis. Curr Med Chem 2010;17:1942-1199.

57. Zhang F, Zhou H, Wilson BC, Shi JS, Hong JS, Gao HM. Fluoxetine protects neurons against microglial activationmediated neurotoxicity. Parkinsonism Relat Disord 2012;18(Suppl. 1):S213-S217.

58. Lim CM, Kim SW, Park JY, Kim C, Yoon SH, Lee JK. Fluoxetine affords robust neuroprotection in the postischemic brain via its anti-inflammatory effect. J Neurosci Res 2009;87:1037-1045.

59. Alme MN, Wibrand K, Dagestad G, Bramham CR. Chronic fluoxetine treatment induces brain region-specific upregulation of genes associated with BDNF-induced long-term potentiation. Neural Plast 2007;2007:26496.

60. Rudick RA, Mi S, Sandrock AW, Jr. LINGO-1 antagonists as therapy for multiple sclerosis: in vitro and in vivo evidence. Expert Opin Biol Ther 2008;8:1561-1570.

61. Mi S, Miller RH, Lee X, et al. LINGO-1 negatively regulates myelination by oligodendrocytes. Nat Neurosci 2005;8:745-751.

62. Yong VW. Prospects of repair in multiple sclerosis. J Neurol Sci 2009;277(Suppl. 1):S16-S18.

63. Cadavid D, Balcer L, Galetta S, et al. Evidence of remyelination with the anti-LINGO-1 monoclonal antibody BIIB033 after acute optic neuritis. The 67th Annual Meeting of the American Academy of Neurology, Wasgington, DC, April 18-25, 2015.

64. Fitzgerald P, Dinan TG. Prolactin and dopamine: what is the connection? A review article. J Psychopharmacol 2008;22:12-19.

65. Phan H, DeReese A, Day AJ, Carvalho M. The dual role of domperidone in gastroparesis and lactation. Int J Pharm Compd 2014;18:203-207.

66. Zhornitsky S, Yong VW, Weiss S, Metz LM. Prolactin in multiple sclerosis. Mult Scler 2013;19:15-23.

67. Zhornitsky S, Johnson TA, Metz LM, Weiss S, Yong VW. Prolactin in combination with interferon-beta reduces disease severity in an animal model of multiple sclerosis. J Neuroinflamm 2015;12:55.

68. Coyle PK. Multiple sclerosis in pregnancy. Continuum (Minneap Minn) 2014;20:42-59.

69. Brines M, Cerami A. Emerging biological roles for erythropoietin in the nervous system. Nat Rev Neurosci 2005;6:484-494.

70. Bartels C, Spate K, Krampe H, Ehrenreich H. Recombinant human erythropoietin: novel strategies for neuroprotective/neuroregenerative treatment of multiple sclerosis. Ther Adv Neurol Disord 2008;1:193-206.

71. Li W, Maeda Y, Yuan RR, Elkabes S, Cook S, Dowling P. Beneficial effect of erythropoietin on experimental allergic encephalomyelitis. Ann Neurol 2004;56:767-777.
72. Ehrenreich H, Fischer B, Norra C, et al. Exploring recombinant human erythropoietin in chronic progressive multiple sclerosis. Brain 2007;130:2577-2588.

73. Radaelli M, Merlini A, Greco R, et al. Autologous bone marrow transplantation for the treatment of multiple sclerosis. Curr Neurol Neurosci Rep 2014;14:478.

74. Harris VK, Sadiq SA. Stem cell therapy in multiple sclerosis: a future perspective. Neurodegener Dis Manag 2015;5:167-170.

75. Fassas A, Anagnostopoulos A, Kazis A, et al. Peripheral blood stem cell transplantation in the treatment of progressive multiple sclerosis: first results of a pilot study. Bone Marrow Transplant 1997;20:631-638.

76. Nash RA, Bowen JD, McSweeney PA, et al. High-dose immunosuppressive therapy and autologous peripheral blood stem cell transplantation for severe multiple sclerosis. Blood 2003;102: 2364-2372.

77. Fassas A, Kimiskidis VK, Sakellari I, et al. Long-term results of stem cell transplantation for MS: a single-center experience. Neurology 2011;76:1066-1070.

78. Nash RA, Hutton GJ, Racke MK, et al. High-dose immunosuppressive therapy and autologous hematopoietic cell transplantation for relapsing-remitting multiple sclerosis (HALT-MS): a 3-year interim report. JAMA Neurol 2015;72:159-169.

79. Connick P, Kolappan M, Crawley C, et al. Autologous mesenchymal stem cells for the treatment of secondary progressive multiple sclerosis: an open-label phase 2a proof-of-concept study. Lancet Neurol 2012;11:150-156.

80. Gibson LC, Hastings SF, McPhee I, et al. The inhibitory profile of Ibudilast against the human phosphodiesterase enzyme family. Eur J Pharmacol 2006;538:39-42.

81. Feng J, Misu T, Fujihara K, et al. Ibudilast, a nonselective phosphodiesterase inhibitor, regulates Th1/Th2 balance and NKT cell subset in multiple sclerosis. Mult Scler 2004;10:494-498.

82. Mizuno T, Kurotani T, Komatsu Y, et al. Neuroprotective role of phosphodiesterase inhibitor ibudilast on neuronal cell death induced by activated microglia. Neuropharmacology 2004;46: 404-411.

83. Barkhof F, Hulst HE, Drulovic J, et al. Ibudilast in relapsingremitting multiple sclerosis: a neuroprotectant? Neurology 2010;74:1033-1040.

84. Jaber S, Polster BM. Idebenone and neuroprotection: antioxidant, pro-oxidant, or electron carrier? J Bioenerg Biomembr 2015;47: $111-118$.

85. Civenni G, Bezzi P, Trotti D, Volterra A, Racagni G. Inhibitory effect of the neuroprotective agent idebenone on arachidonic acid metabolism in astrocytes. Eur J Pharmacol 1999;370:161-167.

86. Fiebiger SM, Bros H, Grobosch T, et al. The antioxidant idebenone fails to prevent or attenuate chronic experimental autoimmune encephalomyelitis in the mouse. J Neuroimmunol 2013;262:66-71.

87. Salinthone S, Yadav V, Bourdette DN, Carr DW. Lipoic acid: a novel therapeutic approach for multiple sclerosis and other chronic inflammatory diseases of the CNS. Endocr Metab Immune Disord Drug Targets 2008;8:132-142.

88. Marracci GH, Jones RE, McKeon GP, Bourdette DN. Alpha lipoic acid inhibits $\mathrm{T}$ cell migration into the spinal cord and suppresses and treats experimental autoimmune encephalomyelitis. J Neuroimmunol 2002;131:104-114.

89. Yadav V, Marracci G, Lovera J, et al. Lipoic acid in multiple sclerosis: a pilot study. Mult Scler 2005;11:159-165.

90. Jope RS, Yuskaitis CJ, Beurel E. Glycogen synthase kinase-3 (GSK3): inflammation, diseases, and therapeutics. Neurochem Res 2007;32:577-595.

91. De Sarno P, Axtell RC, Raman C, Roth KA, Alessi DR, Jope RS. Lithium prevents and ameliorates experimental autoimmune encephalomyelitis. J Immunol 2008;181:338-345. 
92. Dubreuil P, Letard S, Ciufolini M, et al. Masitinib (AB1010), a potent and selective tyrosine kinase inhibitor targeting KIT. PLoS One 2009;4:e7258.

93. Theoharides TC, Kempuraj D, Kourelis T, Manola A. Human mast cells stimulate activated $\mathrm{T}$ cells: implications for multiple sclerosis. Ann N Y Acad Sci 2008;1144:74-82.

94. Ribatti D. The crucial role of mast cells in blood-brain barrier alterations. Exp Cell Res 2015;338:119-125.

95. Vermersch P, Benrabah R, Schmidt N, et al. Masitinib treatment in patients with progressive multiple sclerosis: a randomized pilot study. BMC Neurol 2012;12:36.

96. Girvan RC, Knight DA, O'Loughlin CJ, Hayman CM, Hermans IF, Webster GA. MIS416, a non-toxic microparticle adjuvant derived from Propionibacterium acnes comprising immunostimulatory muramyl dipeptide and bacterial DNA promotes cross-priming and Th1 immunity. Vaccine 2011;29:545-557.

97. White M, Webster G, O'Sullivan D, Stone S, La Flamme AC. Targeting innate receptors with MIS416 reshapes Th responses and suppresses CNS disease in a mouse model of multiple sclerosis. PLoS One 2014;9:e87712.

98. Innate Immunotherapeutics. MIS416. Available at: http://www. innateimmunotherapeutics.com/irm/content/clinical-trials.aspx? RID=307. Accessed June 14, 2015.

99. Lutterotti A, Martin R. Getting specific: monoclonal antibodies in multiple sclerosis. Lancet Neurol 2008;7:538-547.

100. Rommer PS, Dudesek A, Stuve O, Zettl UK. Monoclonal antibodies in treatment of multiple sclerosis. Clin Exp Immunol 2014;175:373-384.

101. Romme Christensen J, Ratzer R, Bornsen L, et al. Natalizumab in progressive MS: results of an open-label, phase 2A, proof-ofconcept trial. Neurology 2014;82:1499-1507.

102. Biogen reports top-line results from phase 3 study evaluating natalizumab in secondary progressive MS. Available at: http:// media.biogen.com. Accessed 13 Dec 2015.

103. Stuve O, Cravens PD, Eagar TN. DNA-based vaccines: the future of multiple sclerosis therapy? Expert Rev Neurother 2008;8:351360

104. Vandenbark AA. TCR peptide vaccination in multiple sclerosis: boosting a deficient natural regulatory network that may involve TCR-specific CD4+CD25+ Treg cells. Curr Drug Targets Inflamm Allergy 2005;4:217-229.

105. Wu Y, Borde M, Heissmeyer V, et al. FOXP3 controls regulatory $\mathrm{T}$ cell function through cooperation with NFAT. Cell 2006;126: 375-387.

106. Vandenbark AA, Culbertson NE, Bartholomew RM, et al. Therapeutic vaccination with a trivalent T-cell receptor (TCR) peptide vaccine restores deficient FoxP3 expression and TCR recognition in subjects with multiple sclerosis. Immunology 2008;123:66-78.

107. Raftopoulos RE, Kapoor R. Neuroprotection for acute optic neuritis - can it work? Mult Scler Relat Disord 2013;2:307-311.

108. Black JA, Liu S, Carrithers M, Carrithers LM, Waxman SG. Exacerbation of experimental autoimmune encephalomyelitis after withdrawal of phenytoin and carbamazepine. Ann Neurol 2007;62:21-33.

109. Morsali D, Bechtold D, Lee W, et al. Safinamide and flecainide protect axons and reduce microglial activation in models of multiple sclerosis. Brain 2013;136:1067-1082.

110. Kapoor R, Furby J, Hayton T, et al. Lamotrigine for neuroprotection in secondary progressive multiple sclerosis: a randomised, double-blind, placebo-controlled, parallel-group trial. Lancet Neurol 2010;9:681-688.

111. Beck RW, Cleary PA, Anderson MM, Jr., et al. A randomized, controlled trial of corticosteroids in the treatment of acute optic neuritis. The Optic Neuritis Study Group. N Engl J Med 1992;326: $581-588$
112. Kolappan M, Henderson AP, Jenkins TM, et al. Assessing structure and function of the afferent visual pathway in multiple sclerosis and associated optic neuritis. J Neurol 2009;256:305-319.

113. Kapoor R, Raftopoulos R, Hickman S, et al. Phenytoin is neuroprotective in acute optic neuritis: results of a phase 2 randomized controlled trial. The 67th Annual Meeting of the American Academy of Neurology, Wasgington, DC, April 18-25, 2015.

114. Bonnan M, Ferrari S, Bertandeau E, et al. Intrathecal rituximab therapy in multiple sclerosis: review of evidence supporting the need for future trials. Curr Drug Targets 2014;15:1205-1214.

115. Montalban X, Hemmer B, Rammohan K, et al. Efficacy and safety of ocrelizumab in primary progressive multiple sclerosis - results of the placebo-controlled, double-blind, Phase III ORATORIO study. Mult Scler 2015 Supplement, p780-808. 29p.

116. van der Most PJ, Dolga AM, Nijholt IM, Luiten PG, Eisel UL. Statins: mechanisms of neuroprotection. Prog Neurobiol 2009;88: 64-75.

117. Weber MS, Youssef S, Dunn SE, et al. Statins in the treatment of central nervous system autoimmune disease. J Neuroimmunol 2006;178:140-148.

118. Neuhaus O, Stuve O, Zamvil SS, Hartung HP. Are statins a treatment option for multiple sclerosis? Lancet Neurol 2004;3:369 371.

119. Pihl-Jensen G, Tsakiri A, Frederiksen JL. Statin treatment in multiple sclerosis: a systematic review and meta-analysis. CNS Drugs 2015;29:277-291.

120. Stuve O, Youssef S, Steinman L, Zamvil SS. Statins as potential therapeutic agents in neuroinflammatory disorders. Curr Opin Neurol 2003;16:393-401.

121. Chataway J, Schuerer N, Alsanousi A, et al. Effect of high-dose simvastatin on brain atrophy and disability in secondary progressive multiple sclerosis (MS-STAT): a randomised, placebo-controlled, phase 2 trial. Lancet 2014;383:2213-2221.

122. Gonzalez-Cabrera PJ, Brown S, Studer SM, Rosen H. S1P signaling: new therapies and opportunities. F1000Prime Rep 2014;6: 109.

123. Novgorodov AS, El-Alwani M, Bielawski J, Obeid LM, Gudz TI Activation of sphingosine-1-phosphate receptor S1P5 inhibits oligodendrocyte progenitor migration. FASEB J 2007;21:15031514.

124. Brana C, Frossard MJ, Pescini Gobert R, Martinier N, Boschert U, Seabrook TJ. Immunohistochemical detection of sphingosine-1phosphate receptor 1 and 5 in human multiple sclerosis lesions. Neuropathol Appl Neurobiol 2014;40:564-578.

125. Soliven B, Miron V, Chun J. The neurobiology of sphingosine 1phosphate signaling and sphingosine 1-phosphate receptor modulators. Neurology 2011;76:S9-S14.

126. Selmaj K, Li DK, Hartung HP, et al. Siponimod for patients with relapsing-remitting multiple sclerosis (BOLD): an adaptive, doseranging, randomised, phase 2 study. Lancet Neurol 2013;12:756767.

127. Kappos L, Bar-Or A, Cree B, et al. Siponimod (BAF312) for the Treatment of Secondary Progressive Multiple Sclerosis: Design of the Phase 3 EXPAND Trial. The 65th Annual Meeting of the American Academy of Neurology, San Diego, CA, March 1623, 2013.

128. Schmitz K, Barthelmes J, Stolz L, Beyer S, Diehl O, Tegeder I. "Disease modifying nutricals" for multiple sclerosis. Pharmacol Ther 2015;148:85-113.

129. Caruana M, Vassallo N. Tea polyphenols in Parkinson's disease. Adv Exp Med Biol 2015;863:117-137.

130. Aktas O, Prozorovski T, Smorodchenko A, et al. Green tea epigallocatechin-3-gallate mediates T cellular NF-kappa B inhibition and exerts neuroprotection in autoimmune encephalomyelitis. J Immunol 2004;173:5794-5800. 
131. Sun Q, Zheng Y, Zhang X, et al. Novel immunoregulatory properties of EGCG on reducing inflammation in EAE. Front Biosci 2013;18:332-342.

132. Hellings N, Raus J, Stinissen P. T-cell-based immunotherapy in multiple sclerosis: induction of regulatory immune networks by Tcell vaccination. Expert Rev Clin Immunol 2006;2:705-716.

133. Vandenbark AA, Abulafia-Lapid R. Autologous T-cell vaccination for multiple sclerosis: a perspective on progress. BioDrugs 2008;22:265-273.

134. Fox E, Wynn D, Cohan S, Rill D, McGuire D, Markowitz C. A randomized clinical trial of autologous T-cell therapy in multiple sclerosis: subset analysis and implications for trial design. Mult Scler 2012;18:843-852.

135. Robinson AP, Harp CT, Noronha A, Miller SD. The experimental autoimmune encephalomyelitis (EAE) model of MS: utility for understanding disease pathophysiology and treatment. Handb Clin Neurol 2014;122:173-189.

136. Levy H, Assaf Y, Frenkel D. Characterization of brain lesions in a mouse model of progressive multiple sclerosis. Exp Neurol 2010;226:148-158.

137. Chataway J, Nicholas R, Todd S, et al. A novel adaptive design strategy increases the efficiency of clinical trials in secondary progressive multiple sclerosis. Mult Scler 2011;17:81-88.

138. Kairalla JA, Coffey CS, Thomann MA, Muller KE. Adaptive trial designs: a review of barriers and opportunities. Trials 2012;13:145.
139. Freidlin B, Korn EL, Gray R, Martin A. Multi-arm clinical trials of new agents: some design considerations. Clin Cancer Res 2008; 14:4368-4371.

140. Vesterinen HM, Connick P, Irvine CM, et al. Drug repurposing: a systematic approach to evaluate candidate oral neuroprotective interventions for secondary progressive multiple sclerosis. PLoS One 2015;10:e0117705.

141. Miller DH. Biomarkers and surrogate outcomes in neurodegenerative disease: lessons from multiple sclerosis. NeuroRx 2004;1: 284-294.

142. Malmestrom C, Haghighi S, Rosengren L, Andersen O, Lycke J. Neurofilament light protein and glial fibrillary acidic protein as biological markers in MS. Neurology 2003;61:1720-1725.

143. Kuhle J, Plattner K, Bestwick JP, et al. A comparative study of CSF neurofilament light and heavy chain protein in MS. Mult Scler 2013;19:1597-1603.

144. Petzold A, Eikelenboom MJ, Gveric D, et al. Markers for different glial cell responses in multiple sclerosis: clinical and pathological correlations. Brain 2002;125:1462-1473.

145. Botas A, Campbell HM, Han X, Maletic-Savatic M. Metabolomics of Neurodegenerative Diseases. Int Rev Neurobiol 2015;122:53-80.

146. Freidlin B, Korn EL. Biomarker-adaptive clinical trial designs. Pharmacogenomics 2010;11:1679-1682. 